Research Paper

\title{
Downregulation of BANCR Promotes Aggressiveness in Papillary Thyroid Cancer via the MAPK and PI3K Pathways
}

\author{
Jinjun Zhang ${ }^{*}$, Yaying $\mathrm{Du}^{1^{*}}$, Xiaoxue Zhang2, Mengchen Li², and Xingrui $\mathrm{Li}^{1}{ }^{\circledR}$ \\ 1. Department of Thyroid and Breast Surgery, Tongji Hospital, Tongji Medical College of Huazhong University of Science and Technology, Wuhan, Hubei \\ 430030, P.R. China \\ 2. Department of Obstetrics and Gynecology, Tongji Hospital, Tongji Medical College, Huazhong University of Science and Technology, Wuhan, Hubei \\ 430030, P.R. China \\ ${ }^{*}$ Equal contributors
}

$\square$ Corresponding author: Xingrui Li, M.D., Ph. D., Department of Thyroid and Breast Surgery, Tongji Hospital, Tongji Medical College of Huazhong University of Science and Technology, Wuhan, Hubei 430030, P.R. China. Telephone: +86-27-83665317; E-mail: lixingrui@tjh.tjmu.edu.cn

(C) Ivyspring International Publisher. This is an open access article distributed under the terms of the Creative Commons Attribution (CC BY-NC) license (https://creativecommons.org/licenses/by-nc/4.0/). See http://ivyspring.com/terms for full terms and conditions.

Received: 2017.03.19; Accepted: 2017.12.02; Published: 2018.03.26

\begin{abstract}
Recent evidence indicates that long non-coding RNAs play important roles in tumorigenesis and cancer progression. BRAF-activated non-protein coding RNA (BANCR) is a novel and potential regulator of cancer cell proliferation and migration. However, little is known regarding the role of $B A N C R$ in papillary thyroid cancer (PTC). The current study used quantitative PCR to demonstrate that BANCR was significantly downregulated in 60 paired PTC tissues compared with normal tissues. In addition, BANCR was significantly correlated with lymph node metastasis $(p=0.02)$. Furthermore, Cell Counting Kits and Transwell assays were used to demonstrate that knocking down BANCR with short hairpin RNA (shRNA) transfection significantly promoted the proliferation and invasion of PTC cell lines. The flow cytometric analysis of apoptosis and the cell cycle revealed that the overexpression of BANCR inhibited cancer cell proliferation and invasion, which was associated with the induction of cell-cycle G2/M phase arrest and increased apoptosis. Moreover, western blotting was used to show that the MAPK and PI3K-Akt pathways were aberrantly activated during $B A N C R$-mediated PTC cell proliferation and migration. These findings revealed that BANCR functions as a tumor suppressor during thyroid carcinogenesis.
\end{abstract}

Key words: papillary thyroid cancer; BRAF-activated non-protein coding RNA; MAPK signaling pathway; PI3K-Akt signaling pathway; cell cycle; apoptosis

\section{Introduction}

Thyroid cancer is a common endocrine malignancy that has rapidly increased in global incidence in recent decades [1-3]. In China, the incidence of thyroid cancer increased by $20.1 \%$ between 2003 and 2011, which was the highest increase among all cancers [4]. Follicular thyroid cell-derived tumors include papillary thyroid cancer (PTC), follicular thyroid cancer (FTC), poorly differentiated thyroid cancer (PDTC), and anaplastic thyroid cancer (ATC) [5]. PTC is the primary component of the rapid rise in the incidence of thyroid cancer; it accounts for $80-90 \%$ of all thyroid cancers [6]. Although PTC is a highly curable and indolent cancer that has excellent prognosis, $10 \%$ of patients with aggressive tumors will develop progressive disease $[7,8]$. Therefore, it is important to understand molecular mechanisms involved in the development and progression of thyroid cancer. Although several molecules contributing to tumor invasion and migration have been identified $[5,9,10]$, the precise mechanisms underlying tumor pathogenesis remain to be fully elucidated. 
Recently, thousands of long non-coding RNAs (lncRNAs) were uncovered by deep sequencing; they have a large variety of roles in both gene expression and remodeling of the eukaryotic genome [11]. LncRNAs are transcripts that are longer than 200 nucleotides, devoid of evident open reading frames, and often polyadenylated. Emerging evidence suggests that lncRNAs play a crucial role in gene regulation, which is strongly associated with cell fate determination and human disease, especially tumorigenesis [12-14]. For example, HOTAIR is highly expressed in $\sim 10 \%$ of breast cancer patients $[15,16]$, MALAT1 is significantly associated with non-small cell lung cancer [17], and the aberrant expression of GAS5 is observed in prostate cancer [18]. Together, these findings suggest that lncRNAs are important for understanding the molecular biology of cancer progression, including thyroid cancer.

$B R A F$-activated non-protein coding RNA (BANCR) is a 693-base pair (bp) lncRNA originally identified using massively parallel cDNA sequencing in melanoma cells. It is highly expressed in melanoma and contributes to cell migration $[19,20]$. Previous studies revealed that $B A N C R$ functions as both an oncogene and a tumor suppressor. Wang et al. demonstrated that BANCR expression was upregulated in six pairs of PTC and matching adjacent normal tissues and that BANCR increased PTC cell proliferation by activating autophagy [21]. Interestingly, Tian et al. reported that BANCR expression was downregulated in $68.5 \%$ (63/92) of PTC tissues compared with normal tissues [22]. However, the significance of $B A N C R$ in thyroid cancer is still unclear. In the present study, we assessed the expression of BANCR and performed in vitro studies to elucidate its role in PTC.

\section{Materials and methods}

\section{Tissue samples}

Tissue was obtained from patients (18 male and 42 female) who underwent surgery at the Department of Thyroid and Breast Surgery, Tongji Hospital, Tongji Medical College of Huazhong University of Science and Technology in 2016. The study was carried out in accordance with the institutional ethical guidelines, and the use of human tissues was approved by the Medical Ethics Committee of Tongji Hospital (Institution Review Board Approval: TJ-C20141222). Each patient involved in the study was asked to sign a written informed consent form and the specimens were anonymized and handled according to accepted ethical and legal standards. The diagnosis of papillary thyroid cancer was confirmed histopathologically by an experienced pathologist. All samples were snap-frozen in RNAlater solution and stored at $-80^{\circ} \mathrm{C}$ until use. Detailed patient descriptions are provided in Supplementary Table 1.

\section{Cell line and culture conditions}

Three human PTC cells lines NPA, BCPAP, TPC1 and normal thyroid tissue cell lines Nthy-ori3-1 were provided by The Institute of Interdisciplinary Research (Berkeley, CA, USA). The cell lines were authenticated at Shanghai Biowing Applied Biotechnology Co. Ltd using short tandem repeat (STR) DNA profiling (ABI 3730XL Genetic Analyzer, Life Technologies, Waltham, MA, USA). The cells were used in experiments within 25 passages. TPC1 and BCPAP cells were maintained in RPMI-1640 medium (Boster, Wuhan, China). NPA and Nthy-ori3-1 cells were maintained in DMEM medium (Boster), both supplemented with 10\% fetal bovine serum (Gibco, Carlsbad, CA, USA), in a $37^{\circ} \mathrm{C}$ incubator with a humidified atmosphere of $5 \% \mathrm{CO}_{2}$.

\section{RNA interference using shRNA}

$B A N C R$ was knocked down by transfecting cells with BANCR short hairpin RNA (shRNA, Genepharma, Shanghai, China) using Lipofectamine 3000 (Invitrogen, Carlsbad, CA, USA). The target sequences were as follows:

LV3-BANCR -323 (shRNA1\#), 5'-GGAGTGGCG ACTATAGCAAAC-3

LV3-BANCR -540 (shRNA2\#), 5'-GGACTCCAT GGCAAACGTTGT-3

BANCR shRNA stable transfectants were selected in medium containing $2 \mu \mathrm{g} / \mathrm{mL}$ puromycin (Goodbio, Wuhan, China). LV3-NC empty vector was used as the control. The specific silencing of BANCR expression was assessed using qRT-PCR.

\section{Plasmid DNA transfection}

The ectopic expression of BANCR was achieved by transfecting cells with pEX-2-BANCR (Genepharma, Shanghai, China) using Lipofectamine 3000. $B A N C R$-overexpressing stable transfectants were selected in medium containing G418 (Goodbio, Wuhan, China; $800 \mu \mathrm{g} / \mathrm{mL}$ for NPA cells and 1100 $\mu \mathrm{g} / \mathrm{mL}$ for TPC1 cells). $\mathrm{pEX}-2-\mathrm{NC}$ empty vector was used as the control. The expression of BANCR was assessed using qRT-PCR.

\section{RNA extraction and quantitative real-time PCR}

Total RNA was extracted from tissues or cells using Trizol reagent (Invitrogen). BANCR expression was then measured in triplicate using SYBR Green qPCR Mix (Toyobo, Shanghai, China). Primer sequences were as follows:

BANCR 
Forward: 5'-ACAGGACTCCATGGCAAACG-3'

Reverse: 5'-ATGAAGAAAGCCTGGTGCAGT-3' GAPDH

Forward: 5'- GGGAGCCAAAAGGGTCAT-3'

Reverse: 5'- GAGTCCTTCCACGATACCAA-3'

The data were processed using the $2-\Delta \Delta \mathrm{CT}$ method and normalized to GAPDH expression.

\section{Cell viability assays}

Cell viability was monitored using Cell Counting Kit-8 (CCK-8, Dojindo, Kyushu, Japan) according to the manufacturer's instructions. NPA and TPC1 cells were seeded into six-well plates (Corning, New York, NY, USA) and incubated overnight. They were then transfected with BANCR shRNA1\# and 2\#, $\mathrm{pEX}-2-B A N C R$, or the respective negative control. The cells were maintained for $48 \mathrm{~h}$ and then seeded into 96-well plates (3000 cells per well). The cells were maintained for $4 \mathrm{~h}$, and CCK- 8 was then added to each well and incubated at $37^{\circ} \mathrm{C}$ for $3 \mathrm{~h}$. The optical densities (ODs) were measured at a wavelength of 450 $\mathrm{nm}$ using a microplate reader. The results are represented as the mean of five different wells; wells were assessed in triplicate for each treatment group.

\section{Transwell migration and invasion assays}

For Transwell invasion assays, $3 \times 10^{4} \mathrm{NPA}$ and TPC1 cells in serum-free medium were plated in the upper chamber that was coated with Matrigel (24-well insert, 8-mm pore size; Corning, NY, USA). For the Transwell migration assays the upper chamber was not coated with Matrigel. The media, which were supplemented with serum, served as a chemotactic agent in the lower chamber. The cells were incubated for $72 \mathrm{~h}$ and $48 \mathrm{~h}$ for invasion and migration assays, respectively. The cells that did not migrate through the pores were removed with a cotton swab, and the cells on the lower surface of the membrane were stained with crystal violet (Goodbio, Wuhan, China). The number of cells that penetrated the membrane was then counted in five random fields per chamber under a microscope (Olympus Corp. Tokyo, Japan). Each experiment was performed in triplicate.

\section{Protein extraction and western blotting}

Western blotting was performed as described previously [23]. The blots were probed with primary antibodies, including those against p-MEK, ERK1/2, p-ERK1/2, EGFR, p-EGFR, p-PI3K, AKT, p-AKT, p-FAK, p-STAT1, and p-STAT3 (all from Cell Signaling Technology, Danvers, MA, USA), followed by HRP-conjugated secondary antibodies. Signals were visualized using ECL reagent (Goodbio, Wuhan, China). The intensity of the bands was quantified using densitometry (Quantity One software; Bio-Rad,
Berkeley, CA, USA); GAPDH was used as a control.

\section{Flow-cytometric analysis of the cell cycle and apoptosis}

pEX-2-BANCR, BANCR shRNA1\# and 2\#, and empty vector-transfected NPA and TPC1 cells were cultured in six-well plates for $48 \mathrm{~h}$, washed with ice-cold phosphate-buffered saline (PBS), and fixed with $75 \%$ ethanol overnight. The cells were then collected by centrifugation (1000 rpm for $5 \mathrm{~min}$ ) and resuspended in $1 \mathrm{ml}$ of propidium iodide (PI) solution (50 $\mathrm{mg} / \mathrm{mL}$ in PBS) containing $0.25 \mathrm{mg} / \mathrm{mL}$ of RNaseA. After incubation for $30 \mathrm{~min}$ in the dark at $4^{\circ} \mathrm{C}$, the cells were analyzed by flow cytometry (FACScan; BD Biosciences, Franklin Lakes, NJ, USA) using an instrument equipped with Cell Quest software (BD Biosciences). The percentage of cells in G0-G1, S, and G2-M phase was counted and compared.

For apoptosis, analysis, pEX-2-BANCR, BANCR shRNA1\# and 2\#, and empty vector-transfected NPA and TPC1 cells were harvested $48 \mathrm{~h}$ after transfection. Floating and adherent cells were collected using $0.1 \%$ trypsin, washed twice with cold PBS, and suspended in $0.5 \mathrm{~mL}$ binding buffer $(10 \mathrm{mmol} / \mathrm{L}$ HEPES buffer $\mathrm{pH} 7.4,140 \mathrm{mmol} / \mathrm{L} \mathrm{NaCl}$, and $2.5 \mathrm{mmol} / \mathrm{L} \mathrm{CaCl} 2$ ). The cells were then treated with an Annexin V-APC/7-AAD Apoptosis Detection Kit (Abnova, Atlanta, GA, USA) according to the manufacturer's instructions. Flow cytometry was carried out using FACScan (BD Biosciences). The percentage of early and late apoptotic cells were compared among groups in each experiment. This assay was repeated three times.

\section{Fluorescence in situ hybridization (FISH)}

Cells were plated onto coverslips, washed with phosphate buffered saline (PBS), fixed in $4 \%$ paraformaldehyde for $10 \mathrm{~min}$, and permeabilized with $0.25 \%$ Triton-100 for 5 min Cy3-labeled lncRNA BANCR probes were obtained from RiboBio (Guangzhou, China). Slides of tissues were hybridized with probes overnight, washed as the above description. RNA FISH was performed using a FISH kit (Ribobio Co., Guangzhou, China) following the manufacturer's instructions. Then, the slides were counterstained with 4, 6-diamidino-2-phenylindole (DAPI) to mark the nuclei, U6 and $18 \mathrm{~S}$ were used as cytoplasmic and nuclear positive control. Fluorescently labeled cells were visualized and imaged under a confocal laser scanning microscope (Olympus Corp. Tokyo, Japan).

\section{Statistical analysis}

Statistical analyses were performed using SPSS software version 21 (SPSS, Inc., Chicago, IL, USA) and 
GraphPad prism 5.0 for Windows (GraphPad Software, La Jolla, CA, USA). The Wilcoxon signed rank tests were applied to assess the expression of $B A N C R$ in cancer tissues compared with paired normal tissues. Chi-square tests or Fisher's exact test were used to examine the relationship between $B A N C R$ expression and clinicopathologic characteristics. The differences among groups in proliferation assays, western blotting, and PCR were estimated using Student's $t$-tests or one-way ANOVA. The results are reported as means \pm SDs. Statistical significance was assigned as $p<0.05\left(^{*}\right)$ or $p<0.01\left(^{* *}\right)$.

Table 1. Correlation between BANCR expression and clinical pathological characteristics in papillary thyroid carcinoma

\begin{tabular}{|c|c|c|c|c|}
\hline Characteristics & $\mathrm{n}$ & High expression (\%) & Low expression (\%) & $P$ \\
\hline Gender & 60 & & & \\
\hline Female & 42 & $11(26.2)$ & $31(73.8)$ & 0.755 \\
\hline Male & 18 & $6(33.3)$ & 12(66.7) & \\
\hline \multicolumn{5}{|l|}{ Age (y) } \\
\hline$<45$ & 35 & $11(31.4)$ & $24(68.6)$ & 0.575 \\
\hline$\geq 45$ & 25 & $6(24.0)$ & $19(76.0)$ & \\
\hline \multicolumn{5}{|l|}{ Clinical stage } \\
\hline I-II & 49 & $16(32.7)$ & $33(67.3)$ & 0.155 \\
\hline III-IV & 11 & $1(9.1)$ & $10(90.9)$ & \\
\hline \multicolumn{5}{|l|}{ Tumor depth } \\
\hline T1-T2 & 55 & $17(30.9)$ & $38(69.1)$ & 0.309 \\
\hline T3-T4 & 5 & $0(0.0)$ & $5(100)$ & \\
\hline \multicolumn{5}{|l|}{ Tumor size } \\
\hline$<1 \mathrm{~cm}$ & 16 & $2(12.5)$ & $14(87.5)$ & 0.122 \\
\hline $1-4 \mathrm{~cm}$ & 27 & 11(40.7) & $16(59.3)$ & \\
\hline$>4 \mathrm{~cm}$ & 17 & $4(23.5)$ & $13(76.5)$ & \\
\hline \multicolumn{5}{|l|}{$\begin{array}{l}\text { Lymph node } \\
\text { metastasis }\end{array}$} \\
\hline $\mathrm{Y}$ & 36 & $6(16.7)$ & $30(83.3)$ & $0.020^{*}$ \\
\hline $\mathrm{N}$ & 24 & $11(45.8)$ & $13(54.2)$ & \\
\hline NIA & 20 & $4(20.0)$ & $16(80.0)$ & $0.043^{*}$ \\
\hline NIB & 16 & $2(12.5)$ & 14(87.5) & \\
\hline
\end{tabular}

\section{Results}

\section{Expression of BANCR in PTC tissues and PTC cell lines}

qRT-PCR was used to examine BANCR levels in 60 PTC tissues and matched normal thyroid tissues. The expression of BANCR was significantly downregulated $(p<0.01)$ in $71.7 \%(43 / 60)$ of cancerous tissues compared with normal tissues (Figure 1A). Furthermore, the correlation between BANCR expression and the patients' clinicopathological parameters (including gender, age, and TNM stage) was assessed to determine its clinical significance. BANCR expression in PTC was significantly correlated with lymph node metastasis $(p$ $=0.02)$. BANCR expression was lower in $83.3 \%$ $(30 / 36)$ of the patients with lymph node metastasis (Table 1). Two patients with advanced disease, one with invasion into subcutaneous soft tissues and the other with invasion into the larynx (Supplementary Table 1), both had lower BANCR expression. However, BANCR expression was not associated with other PTC parameters such as gender $(p=0.755)$, tumor stage $(p=0.155)$, and patient age $(p=0.575)$ (Table 1). The clinicopathological parameters for all patients were summarized in Supplementary Table 1. These analyses demonstrate that BANCR may be a good diagnostic biomarker for PTC.

$B A N C R$ expression was also detected in two human PTC cell lines (NPA and TPC1) using qRT-PCR. The two cell lines exhibited a notable downregulation in BANCR compared with control thyroid tissues (Figure 1B, $p=0.024$ ). In the FISH experiment, which involved PTC cell lines (BCPAP and TPC1) and PTC patient specimens, we also confirmed the decreased BANCR expression, which further suggested that lncRNA BANCR was mainly localized in the cytoplasm of PTC cells and tissues (Figure $1 \mathrm{~F}$ and $1 \mathrm{G}$ ).

\section{Modulating BANCR expression affects cell proliferation and motility}

To clarify the role of BANCR in PTC cells, CCK-8 was used to assess the impact of BANCR overexpression or knockdown on NPA and TPC1 cells. Our clinical data indicated that BANCR expression was inversely correlated with tumor size and lymph node metastasis. Thus, the shRNAs LV3-BANCR-323 (shRNA1\#) and LV3-BANCR-540 (shRNA2\#) were used to downregulate endogenous BANCR expression in NPA and TPC1 cells. CCK-8 assays were then performed to monitor the effects of $B A N C R$ shRNA on cell proliferation. The results showed that the proliferation of BANCR shRNAtransfected was higher than control cells transfected with the empty vector at $48 \mathrm{~h}(p<0.01$; Figure $2 \mathrm{~A}$ and 2B). Transwell migration and invasion assays showed that downregulating BANCR significantly promoted cell motility $(p<0.01$; Figure $2 \mathrm{C}$ and $2 \mathrm{D})$.

Consistent with these observations, the growth of NPA and TPC1 cells transfected with pEX-2-BANCR was impaired compared with control cells $(p<0.01$; Figure $2 \mathrm{~A}$ and 2B). To further examine the effects of BANCR on the motility of NPA and TPC1 cells, transwell migration and invasion assays were performed. BANCR overexpression impaired the migration and invasion of PTC cells $(p<0.01$; Figure $2 \mathrm{E}$ and $2 \mathrm{~F}$ ), suggesting that BANCR is at least partially necessary for PTC cell motility in vitro. These observations suggest that BANCR contributes to PTC progression by enhancing cell proliferation and motility. 
A

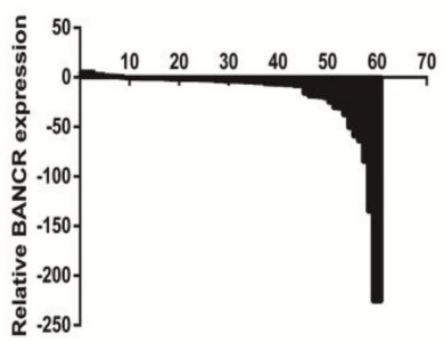

$\mathrm{C}$

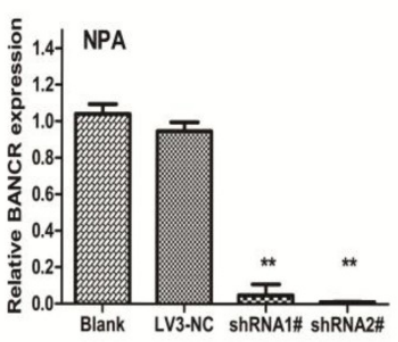

D

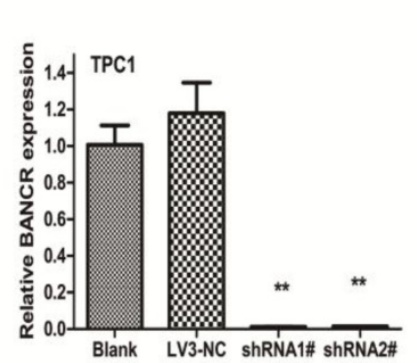

B

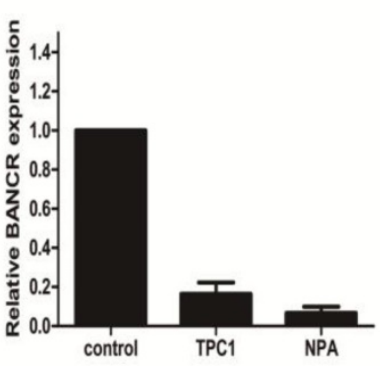

E

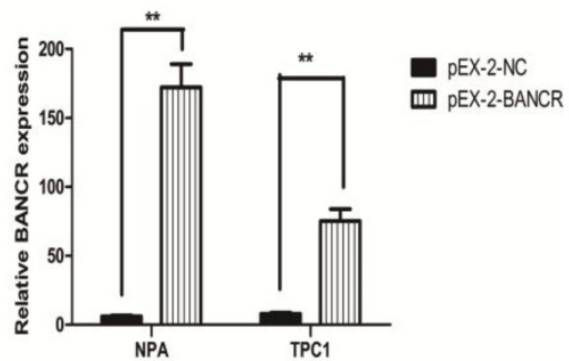

F

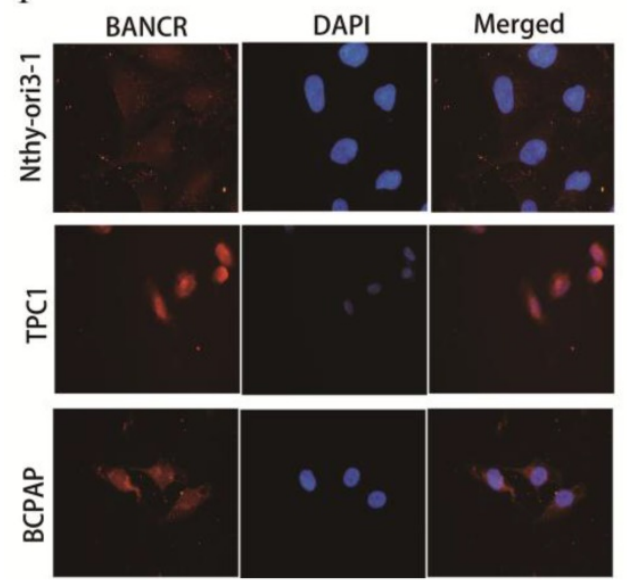

G
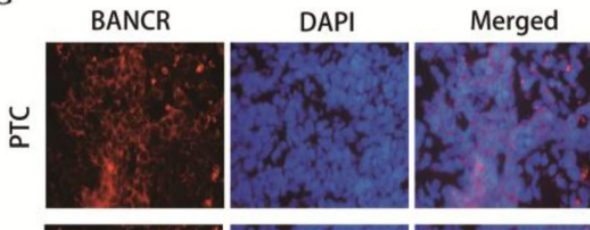

흘
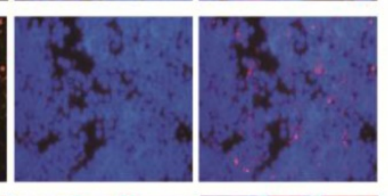

흠
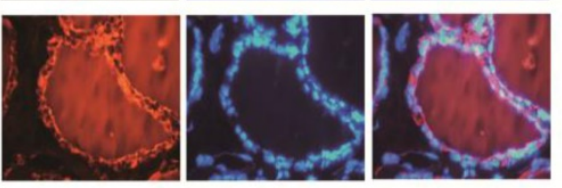

Figure 1. Relative expression of BANCR in papillary thyroid cancer tissues and cell lines. (A) Relative expression of BANCR in papillary thyroid cancer tissues $(n=60)$ compared with corresponding normal tissues $(n=60)$. BANCR expression was examined by qPCR and normalized to GAPDH expression. The results are presented as the fold-change in tumor tissues relative to normal tissues. (B) BANCR expression in NPA and TPCl papillary thyroid cancer cell lines compared with control thyroid tissues pooled from three controls. (C and D) qPCR analysis of BANCR expression following the treatment of NPA and TPCl cells with BANCRshRNAI\#, shRNA2\#, and empty vector. (E) qPCR analysis of BANCR expression following the treatment of NPA and TPCI cells with pEX-2-BANCRand empty vector.(F) Fluorescence in situ hybridization in Nthy-ori3-1, TPCI and BCPAP cells (G) Fluorescence in situ hybridization in papillary thyroid cancer, metastatic lymph node and normal thyroid tissues. **, $\mathrm{p}<0.01$. shRNAI\#, shRNA2\#, and LV3-NC, plasmid containing shRNAI\# and shRNA2\# for BANCR and negative control shRNA, respectively; pEX-2-BANCRand pEX-2-NC, pEX-2 plasmid containing human full-length BANCR cDNA and negative control, respectively; BANCR, BRAF-activated non-protein coding RNA; shRNA, short hairpin RNA; DAPI, 4',6-diamidino-2-phenylindole.

\section{BANCR promotes G2/M arrest and causes apoptosis}

To determine whether the effects of BANCR on the proliferation and motility of PTC cells were mediated by inhibiting cell cycle progression, flow cytometry was used to assess the cell cycle in NPA cells (Figure $3 \mathrm{~A}$ and $3 \mathrm{~B}$ ). BANCR overexpression led to the significant accumulation of cells in G2/M-phase and a significant decrease in cells in S-phase compared with control (both $p<0.05$ ). Conversely, knocking down BANCR led to a significant decrease in the percent of cells in G1/G0- and G2/M-phases and a significant increase in S-phase compared with control (both $p<0.01$ ). Next, the effects of BANCR on apoptosis were assessed in NPA cells (Figure 3C and 3D). The percentage of cells undergoing late apoptosis and necrosis was significantly increased in the $B A N C R$ overexpression group compared with control $(p<0.01)$. The percentage of early apoptotic cells was significantly decreased in the BANCR shRNA group compared with control $(p<0.01)$. Taken together, these data suggest that $B A N C R$ overexpression could induce $\mathrm{G} 2 / \mathrm{M}$ phase arrest and enhance apoptosis in PTC cells, whereas BANCR downregulation promotes the entry of PTC cells into $S$ phase and protects from early apoptosis. 


\section{BANCR regulates $\mathrm{PTC}$ proliferation and migration by inactivating the MAPK and PI3K-Akt pathways}

Next, the activation of the MAPK and PI3K-Akt pathways was assessed using western blotting to explore the potential mechanism underlying the role of BANCR in cell proliferation and migration. BANCR downregulation activated the MAPK (Figure 4) and PI3K-Akt (Figure 5) pathways in NPA and TPC1 cells.

A

Cells viability for $48 \mathrm{~h}$

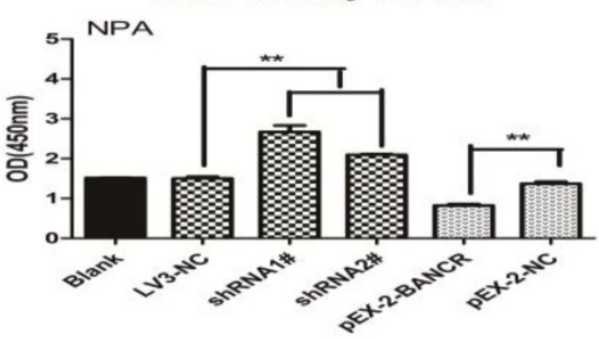

C

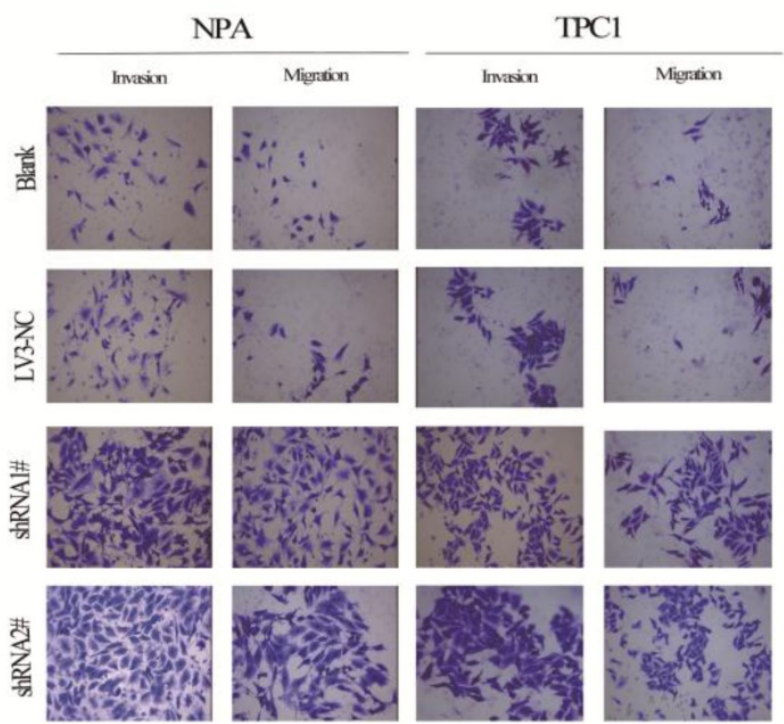

E

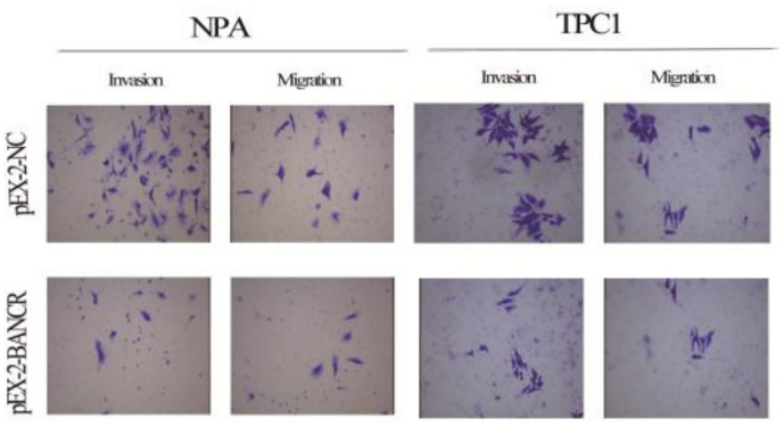

Interestingly, although the MAPK pathway was activated by BANCR knockdown, there was no effect on upstream EGFR or the classical activator MEK1/2; only ERK1/2 phosphorylation was upregulated (Figure 4A and 4C). There was also no difference in the phosphorylation of STAT-1 or STAT-3 (Figure 4E). When BANCR was upregulated, no significant differences were observed in MAPK pathway through either cell line (Fig 4B, 4D and 4F).

B

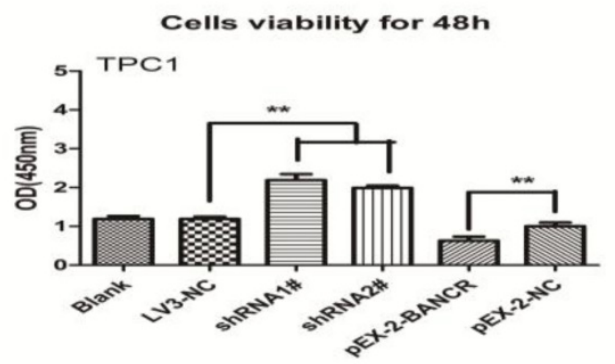

D

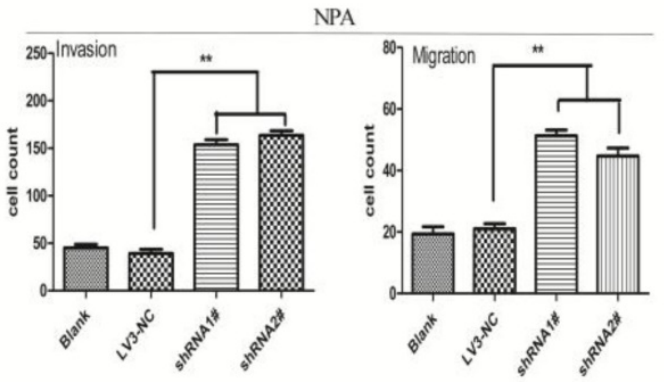

TPCl

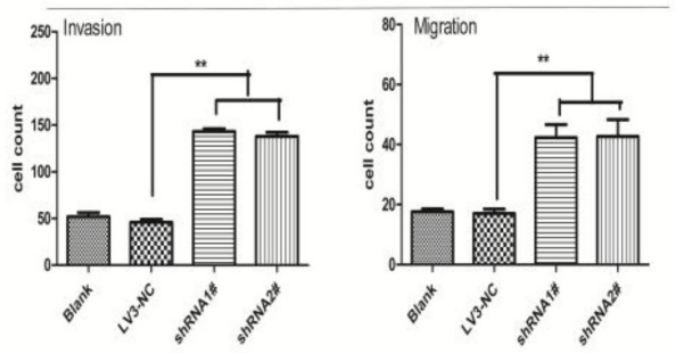

F

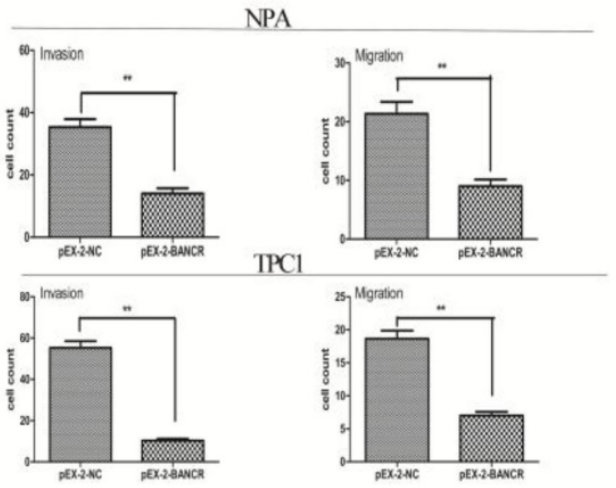

Figure 2. Effects of BANCR on papillary thyroid cancer cell proliferation and motility. (A and B) CCK-8 assays were performed to measure NPA and TPCl cell proliferation. The data represent the means \pm SDs of three independent experiments. ( $C$ and $E$ ) Transwell migration and invasion assays showed that knocking down BANCR promoted NPA and TPCI cell motility. However, cell motility was reduced in BANCR-overexpressing NPA and TPCI cells (crystal violet stain; magnification, $\times 200)$. $(\mathrm{D}$ and $\mathrm{F})$ The bar graph represents at least three independent experiments and the bars indicate the number of migrated cells per field. **p $<0.01$. shRNAI\#, shRNA2\#, LV3-NC respective plasmid containing shRNA for BANCR and negative control;pEX-2-BANCRand pEX-2-NC,pEX-2plasmid containing human full-length BANCR cDNA and negative control, respectively; BANCR, BRAF-activated non-protein coding RNA; shRNA, short hairpin RNA. 
A
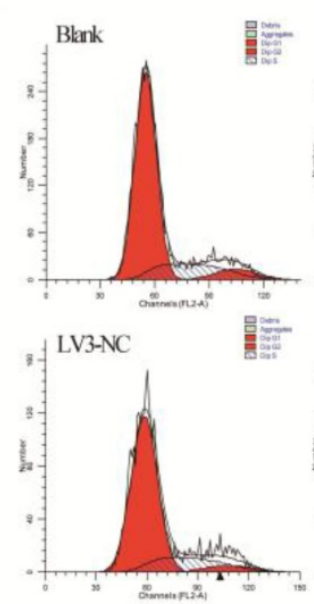

$\mathrm{C}$

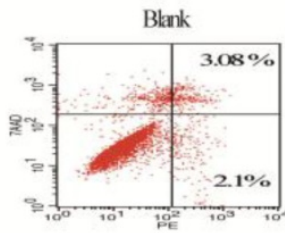

LV3-NC

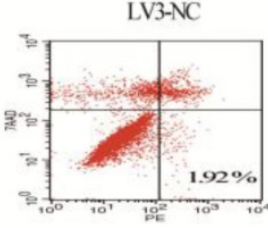

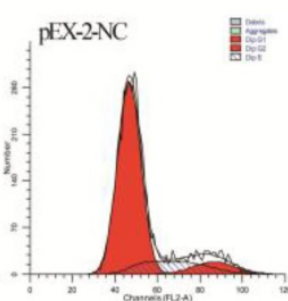
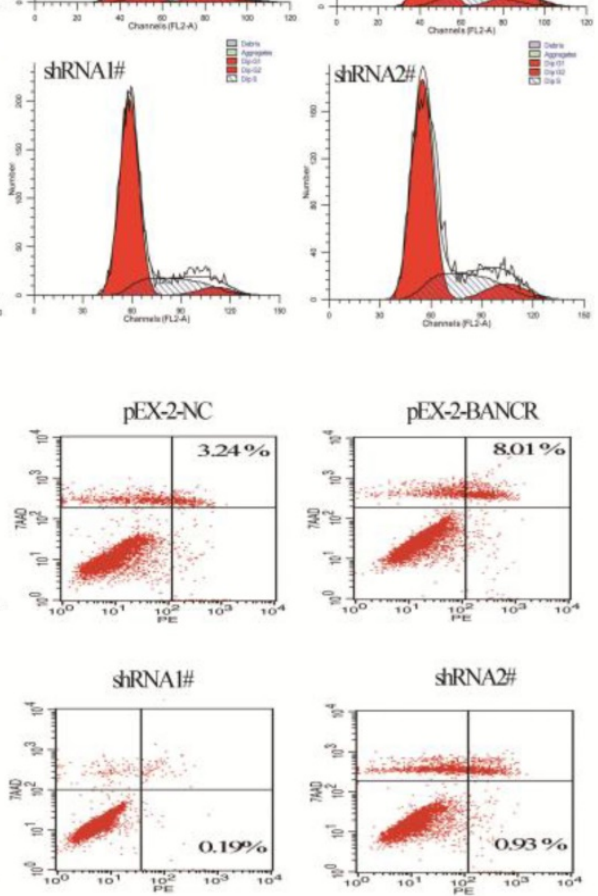
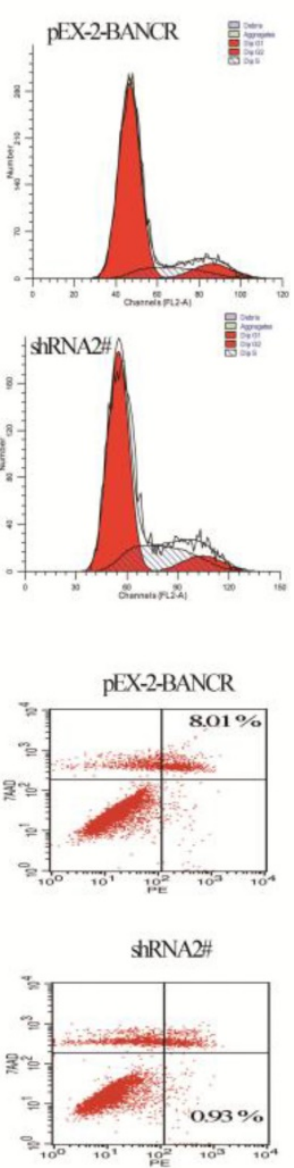

B
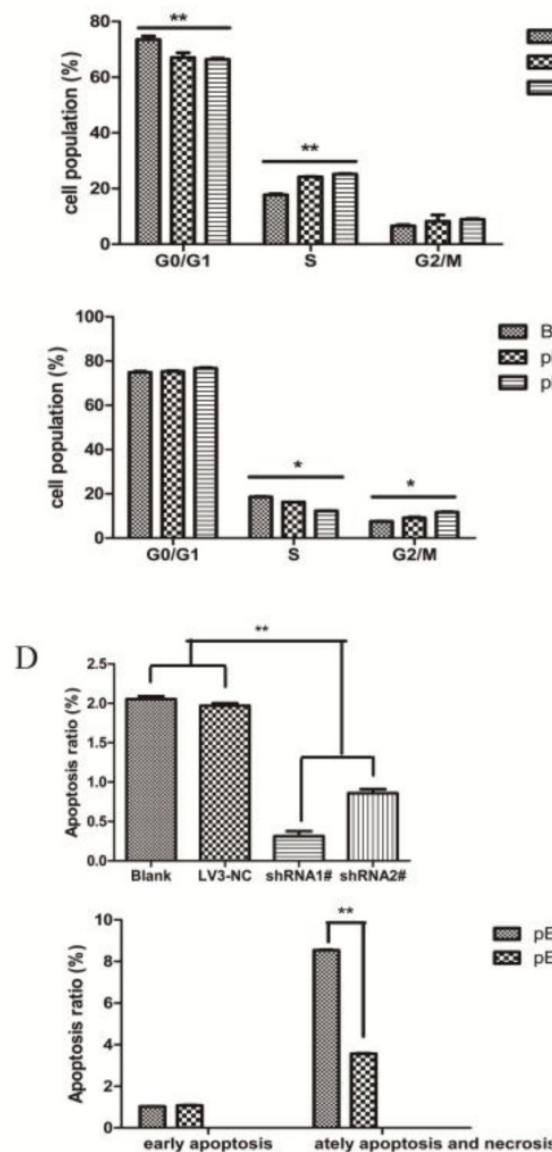

Figure 3. The effects of BANCR on the cell cycle and apoptosis in papillary thyroid cancer cells in vitro. (A and B) The bar chart represents the percentage of cells in G0/G1, S, or G2/M phase. (C and D) The percentage of apoptotic cells was determined by flow cytometry. The data represent the means \pm SDs of three independent experiments. *, $\mathrm{p}<0.05$; **, $\mathrm{p}<0.01$. shRNA1\#, shRNA2\#, LV3-NC respective plasmid containing shRNA for BANCR and negative control; PEX-2 BANCR, pEX-2-NC respective plasmid containing human full length BANCR cDNA and negative control; BANCR, BRAF-activated non-protein coding RNA; shRNA, short hairpin RNA.

The PI3K-Akt pathway was activated by BANCR knockdown, as revealed by increased PI3K and Akt phosphorylation, but there was no effect on FAK (Figure 5A and 5C). In contrast, the PI3K-Akt pathway was inactivated when BANCR was upregulated in NPA cells. Although there were no significant differences in TPC1 cells, while was a similar a trend (Figure 5B and 5D).

\section{Discussion}

Genomic studies have demonstrated that $<2 \%$ of the total human genome can be transcribed, and that noncoding RNAs likely account for the greater complexity transcriptome in eukaryotes [24, 25]. lncRNAs were once neglected and considered to be noise in cells for a long time. However, accumulating evidence has assigned new functions to lncRNAs, including roles in the transcriptional, epigenetic, and post-transcriptional regulation of gene expression [26-28]. It was reported that lncRNAs affect many cellular processes in tumorigenesis such as survival, proliferation, apoptosis, the cell cycle, and migration
[29, 30]. Unlike microRNAs, lncRNAs are not currently well understood. BANCR, a recently found lncRNA, can regulate proliferation and migration in melanoma, colorectal cancer, and lung cancer $[19,31,32]$. In a recent meta-analysis [20], high BANCR expression was correlated with lymph node metastasis, tumor stage, and poor prognosis in gastrointestinal cancer patients, but not in other cancers. However, little is known about the role of $B A N C R$ in the development of papillary thyroid carcinoma; the mechanism underlying tumorigenesis and its clinical significance is still unclear. According to previous studies, the function of BANCR in PTC is controversial. Liao et al. demonstrated that BANCR expression was downregulated in 92 pairs of PTC tissues, as well as in the cell lines TPC-1, K1, and BCPAP. In other studies, Zheng et al. and Wang et al. demonstrated that BANCR is upregulated in human PTC tissues and IHH4 cells and that this promotes cell growth and proliferation [33]. In the current study, we investigated the clinical significance of BANCR and its function and molecular mechanism in PTC. Our 
results revealed that $B A N C R$ levels were significantly reduced in 60 pairs of PTC tissues from patients and PTC cell lines (TPC1 and NPA). The discrepancy with our results may stem from differences in the numbers of tissue samples tested (60 in the current study, 92 in Liao et al., 40 in Zheng et al., and six in Wang et al.), tumor heterogeneity (especially in Zheng et al., who had only 21 cases of classical PTC among 40 samples) and in the BANCR expression patterns in different cell lines (Zheng et al. and Wang et al. used IHH4 cells). In the current study, low BANCR expression levels were significantly correlated with lymph node metastasis, whereas Liao et al. reported that downregulated $B A N C R$ expression was correlated with tumor size, multifocal lesions, and an advanced pathological stage; this difference may stem from tumor heterogeneity. Overall, both studies confirm that low $B A N C R$ levels are associated with poor outcomes in PTC patients.

Liao et al. reported that the overexpression of BANCR promotes apoptosis in TPC1 and $\mathrm{K} 1$ cell lines. However, the current study reported that BANCR overexpression could induce G2/M phase arrest and

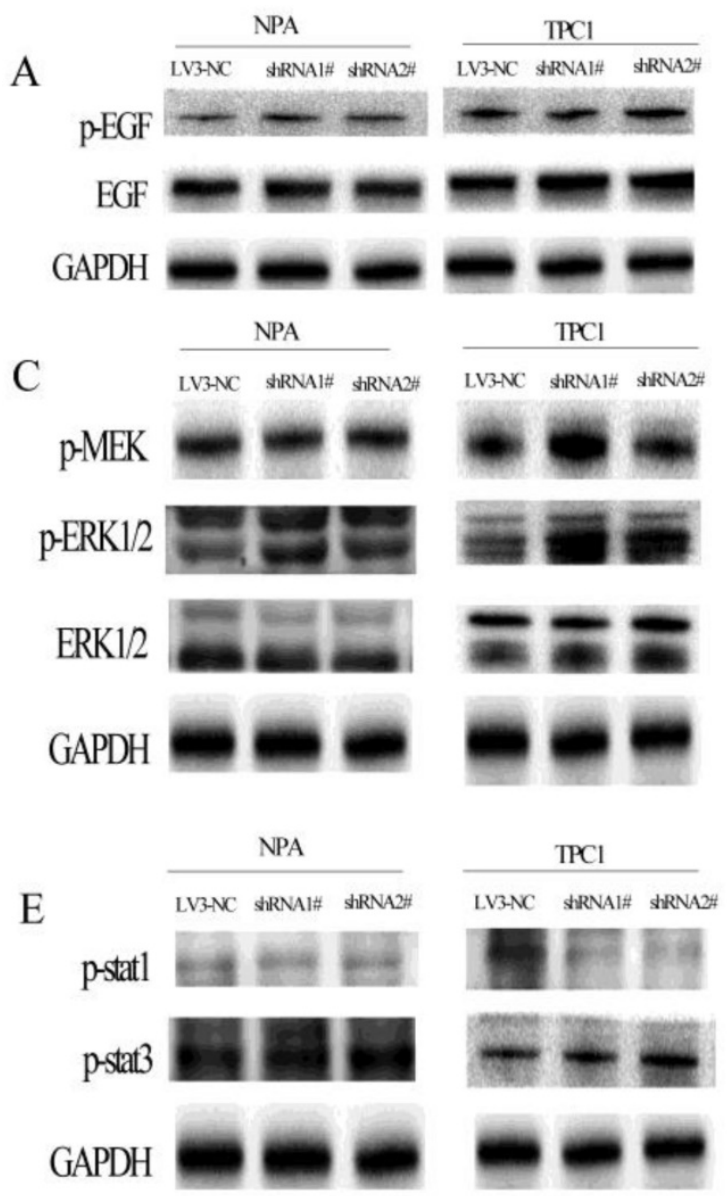

enhance apoptosis in PTC cells whereas BANCR downregulation promoted the entry of PTC cells into $S$ phase and protected from early apoptosis.

The MAPK and PI3K-Akt signaling pathways play important roles in complex cellular programs including proliferation, survival, and migration [34, 35]. The MAPK and PI3K-Akt pathways are involved in differentiated PTC, and the simultaneous activation of both pathways becomes more frequent as the grade of thyroid tumors increases [36-38]. Activation of the MAPK and PI3K-Akt pathways is an important mechanism that drives the progression of thyroid cancer. The current study investigated the activation of the MAPK and PI3K-Akt pathways in PTC cells by measuring the phosphorylation of proteins in the MAPK and PI3K-Akt pathways, including EGFR, MEK, ERK1/2, STAT-1, STAT-3, FAK, AKT, PI3K, in $B A N C R$ overexpressing and knockdown PTC cells. MAPK and PI3K-Akt were activated in BANCR knockdown PTC cells, but the phosphorylation of EGFR, MEK1/2, STAT-1, STAT-3, and FAK was not affected. The overexpression of BANCR by transfection with pEX-2-BANCR inhibited the activation of the
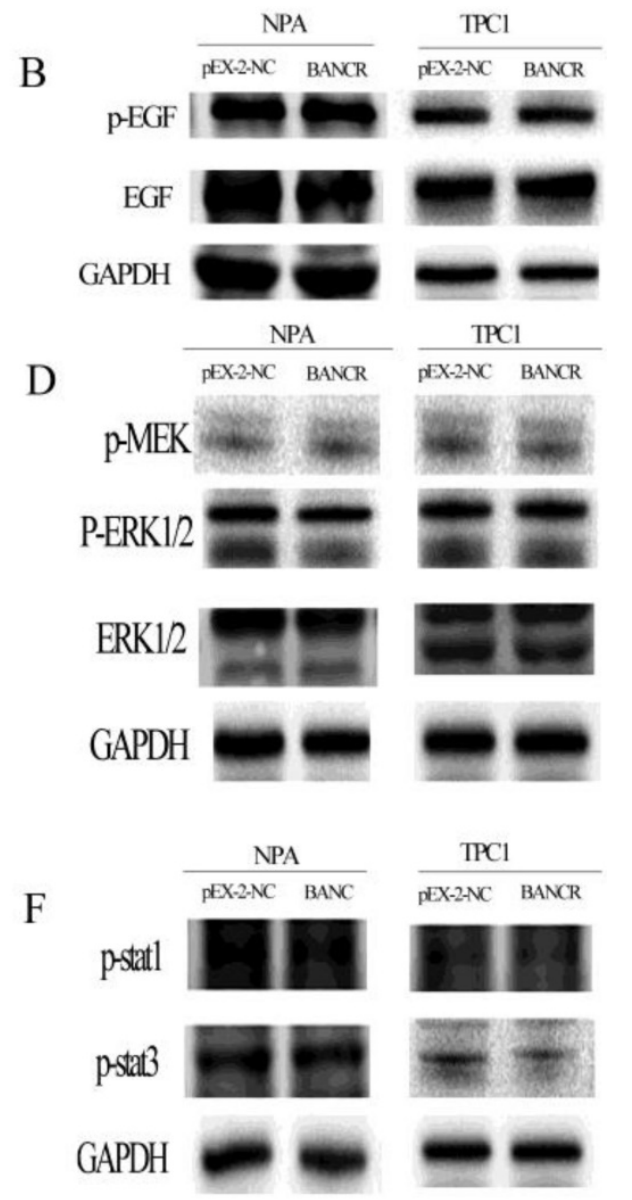

Figure 4. The phosphorylation of EGFR, MEK, ERK1/2, STAT-1, and STAT-3 in BANCR-silenced or -overexpressing NPA and TPC1 cells was detected by western blotting. The loss of BANCR induced the phosphorylation of ERK1/2 (C). However, there was no activation of upstream molecule including EGFR (A), MEK (C), MEK, STAT-1, and STAT-3 (E). BANCR overexpression had no effect on the phosphorylation of EGFR, MEK, ERK1/2, STAT-1, and STAT-3 in either cell line (B, D and F). GAPDH expression was used to normalize protein loading. 
A

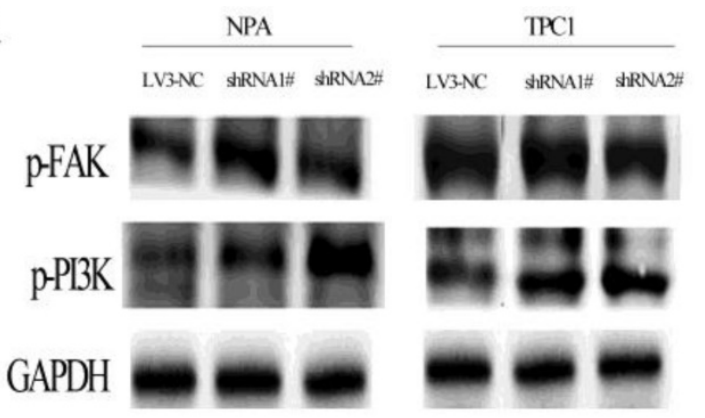

C

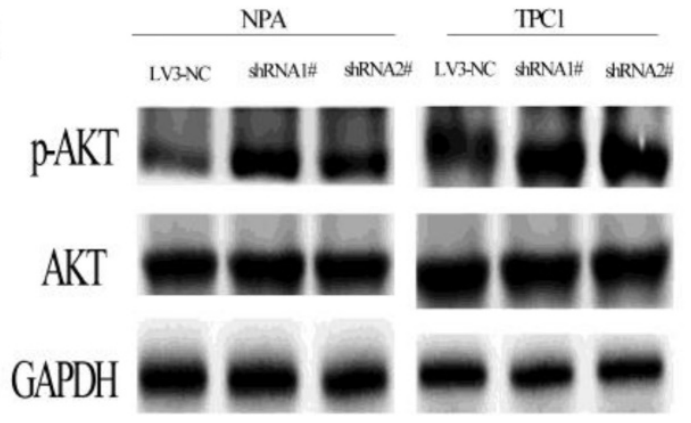

B
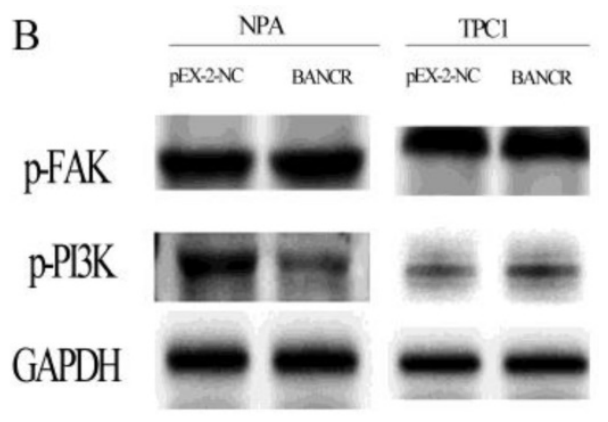

D

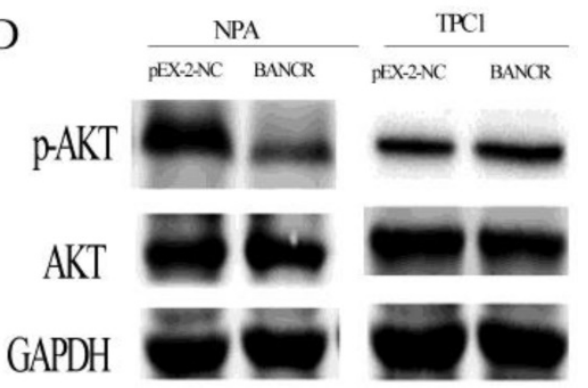

Figure 5. The phosphorylation of FAK, PI3K, and AKT in BANCR-silenced or overexpressing NPA and TPCI cells was detected by western blotting. The loss of BANCR induced the phosphorylation of PI3K (A) and Akt (C). However, there was no activation of the upstream molecule FAK (A). In NPA cells, BANCR overexpression induced the inactivation of PI3K (B) and Akt (D), but there was no activation of the upstream molecule FAK. However, in TPC1 cells BANCR overexpression had no effect on the phosphorylation of FAK, PI3K, and Akt. (B, D) GAPDH expression was used to normalize protein loading.

PI3K-AKT pathway in NPA cells (which contain $\left.B R A F^{\mathrm{V} 600 \mathrm{E}}\right)$, whereas there was no significant difference in the MAPK pathway. Liao et al., demonstrated $B A N C R$ overexpression inactivates ERK1/2 and p38 in K1 cells (which contain BRAF $600 \mathrm{E}$ and PI3K [E542K]). In contrast, the current study found that BANCR knock down increased p44 ERK1 and p42 ERK2 MAPK levels but did not affect the upstream molecules. In addition, the PI3K-Akt pathway was activated by BANCR knockdown and inactivated when $B A N C R$ was upregulated in NPA cells. The discrepancy between these observations and the current results may stem from the different cell lines used. Mammalian cells contain four well-characterized and widely studied MAPKs.

The terminal serine/threonine kinases (MAPKs) include ERK1/2, c-Jun amino-terminal kinases (JNK-12/3; also called SAPKs), p38 kinases (p38a/b/g/d), and ERK5. In the current study, we were most concerned with the EGFR-Ras-Raf-MEKERK MAPK signaling pathway because previous studies revealed that BRAF mutation activate MAPK signaling pathway in thyroid cancer. When BANCR was knocked down with shRNA, only p44 ERK1 and p42 ERK2 MAPKs were increased; the upstream molecules were not affected. When BANCR was overexpressed, the MAPK signaling pathway was not inactivated, as previous studies show that BANCR can recruit zeste homolog $2(\mathrm{EZH} 2)$ to enhance thyroid stimulating hormone receptor which can activate MAPK pathway, we speculate may be this affect the final activation of this pathway. The PI3K-Akt pathway was activated by BANCR knockdown, as represented by increased PI3K and Akt phosphorylation. In contrast, the PI3K-Akt pathway was inactivated when $B A N C R$ was upregulated in NPA but not TPC1 cells. TPC1 cells are a thyroid cancer cell line that contain a RET/PTC rearrangement that can activate the PI3K-Akt pathway; therefore, these complex observations may stem from the different gene expression profiles in the two cancer cell lines. Therefore, we concluded that the effects of BANCR on the proliferation and migration of PTC cells were exerted via the ERK MAPK and PI3K-Akt activations pathways.

Clinical studies performed in BRAF-mutant metastatic melanoma patients treated with selective $B R A F^{\mathrm{V} 600 \mathrm{E}}$ inhibitors treatment revealed that some patients eventually relapse and succumb to acquire chemoresistance via mechanisms involving the reactivation of MAPK signaling [39-41]. Because $B A N C R$ was upregulated by the BRAFV600E mutation, the effects of $B A N C R$ on the MAPK pathway provide a novel mechanism behind the regulation of $B R A F^{\mathrm{V} 600 \mathrm{E}}$ mutations in PTC. These findings will help us fully understand the oncogenesis of PTC.

In summary, the current study demonstrated that BANCR is downregulated in PTC tissues. BANCR 
expression in PTC was significantly correlated lymph node metastasis. The effects of BANCR on cell proliferation and migration were mediated by inducing cell cycle arrest in G2/M phase and inducing apoptosis. Finally, BANCR regulated cell proliferation by activating the ERK-MAPK and PI3K-Akt pathways. Taken together, these findings suggest that BANCR plays a role in the oncogenic process; it may be both a new potential target and prognostic factor for PTC. Further investigations are required to investigate this in more detail.

\section{Abbreviations}

$B A N C R, B R A F$-activated non-protein coding RNA; PTC, papillary thyroid cancer, lncRNAs, long non-coding RNAs; shRNA, short hairpin RNA; FTC, follicular thyroid cancer; PDTC, poorly differentiated thyroid cancer; ATC, anaplastic thyroid cancer; HOTAIR, HOX transcript antisense RNA; MALAT1, metastasis associated lung adenocarcinoma transcript 1; GAS5, growth arrest specific 5; STR, short tandem repeat; MAPK, mitogen activated protein kinase; PI3K, phosphatidylinositol 3'-kinase.

\section{Supplementary Material}

Supplementary figure and table. http://www.jcancer.org/v09p1318s1.pdf

\section{Acknowledgements}

This research was supported by Clinical Research Physician Program of Tongji Medical College, HUST (5001540018).

\section{Conflict of interest}

The authors declare that there is no conflict of interest that could be perceived as prejudicing the impartiality of the research reported.

\section{References}

1. DeSantis CE, Siegel RL, Sauer AG, Miller KD, Fedewa SA, Alcaraz KI and Jemal A. Cancer statistics for African Americans, 2016: Progress and opportunities in reducing racial disparities. CA: A Cancer Journal for Clinicians. 2016; 66(4):290-308.

2. Mao $Y$ and $X i n g ~ M$. Recent incidences and differential trends of thyroid cancer in the USA. Endocr Relat Cancer. 2016; 23(4):313-322.

3. Pellegriti G, Frasca F, Regalbuto C, Squatrito S and Vigneri R. Worldwide increasing incidence of thyroid cancer: update on epidemiology and risk factors. J Cancer Epidemiol. 2013; 2013.965212.

4. Chen W, Zheng R, Baade PD, Zhang S, Zeng H, Bray F, Jemal A, Yu XQ and He J. Cancer statistics in China, 2015. CA: A Cancer Journal for Clinicians. 2016; 66(2):115-132.

5. Xing M. Molecular pathogenesis and mechanisms of thyroid cancer. NAT REV CANCER. 2013; 13(3):184-199.

6. Howlader N, Yu M. 2015 SEER Cancer Statistics Review, National Cancer Institute. Bethesda, MD, 2015\% 1975-2012. http://seer.cancer.gov/csr/1975-2012/. 2015.

7. Brown RL, de Souza JA and Cohen EE. Thyroid cancer: burden of illness and management of disease. J CANCER. 2011; 2:193-199.

8. Tuttle RM, Ball DW, Byrd D, Dilawari RA, Doherty GM, Duh QY, Ehya H, Farrar WB, Haddad RI, Kandeel F, Kloos RT, Kopp P, Lamonica DM, Loree TR, Lydiatt WM and McCaffrey JC, et al. Thyroid carcinoma. J Natl Compr Canc Netw. 2010; 8(11):1228-1274
9. Xing M, Haugen BR and Schlumberger M. Progress in molecular-based management of differentiated thyroid cancer. LANCET. 2013; 381(9871):1058-1069.

10. Agrawal N, Akbani R, Aksoy BA, Ally A and Arachchi H. Integrated Genomic Characterization of Papillary Thyroid Carcinoma. CELL. 2014; 159(3):676-690.

11. Guttman M, Amit I, Garber M, French C, Lin MF, Feldser D, Huarte M, Zuk O, Carey BW, Cassady JP, Cabili MN, Jaenisch R, Mikkelsen TS, Jacks T, Hacohen $\mathrm{N}$ and Bernstein $\mathrm{BE}$, et al. Chromatin signature reveals over a thousand highly conserved large non-coding RNAs in mammals. NATURE. 2009; 458(7235):223-227

12. Guttman M, Donaghey J, Carey BW, Garber M, Grenier JK, Munson G, Young G, Lucas AB, Ach R, Bruhn L, Yang X, Amit I, Meissner A, Regev A, Rinn JL and Root DE, et al. lincRNAs act in the circuitry controlling pluripotency and differentiation. NATURE. 2011; 477(7364):295-300.

13. Wapinski $\mathrm{O}$ and Chang HY. Long noncoding RNAs and human disease. TRENDS CELL BIOL. 2011; 21(6):354-361.

14. Guttman M and Rinn JL. Modular regulatory principles of large non-coding RNAs. NATURE. 2012; 482(7385):339-346.

15. Wan $Y$ and Chang HY. HOTAIR: Flight of noncoding RNAs in cancer metastasis. CELL CYCLE. 2010; 9(17):3391-3392.

16. Gokmen-Polar Y, Vladislav IT, Neelamraju Y, Janga SC and Badve S. Prognostic impact of HOTAIR expression is restricted to ER-negative breast cancers. Sci Rep. 2015; 5:8765.

17. Wu Y, Huang $C$, Meng $X$ and Li J. Long Noncoding RNA MALAT1: Insights into its Biogenesis and Implications in Human Disease. Curr Pharm Des. 2015; 21(34):5017-5028

18. Yacqub-Usman K, Pickard MR and Williams GT. Reciprocal regulation of GAS5 lncRNA levels and mTOR inhibitor action in prostate cancer cells. PROSTATE. 2015; 75(7):693-705

19. Flockhart RJ, Webster DE, Qu K, Mascarenhas N, Kovalski J, Kretz M and Khavari PA. BRAFV600E remodels the melanocyte transcriptome and induces BANCR to regulate melanoma cell migration. GENOME RES. 2012; 22(6):1006-1014.

20. Fan $\mathrm{YH}, \mathrm{Ye} \mathrm{MH}, \mathrm{Wu} \mathrm{L}, \mathrm{Wu}$ MJ, Lu SG and Zhu XG. BRAF-activated lncRNA predicts gastrointestinal cancer patient prognosis: a meta-analysis. ONCOTARGET. 2017; 8(4):6295-6303.

21. Wang Y, Guo Q, Zhao Y, Chen J, Wang S, Jun HU and Sun Y. BRAF-activated long non-coding RNA contributes to cell proliferation and activates autophagy in papillary thyroid carcinoma. ONCOL LETT. 2014; 8(5):1947-1952.

22. Liao T, Qu N, Shi RL, Guo K, Ma B, Cao YM, Xiang J, Lu ZW, Zhu YX, Li DS and Ji QH. BRAF-activated LncRNA functions as a tumor suppressor in papillary thyroid cancer. ONCOTARGET. 2017; 8(1):238-247.

23. Sun M, Liu XH, Wang KM, Nie FQ, Kong R, Yang JS, Xia R, Xu TP, Jin FY, Liu ZJ, Chen JF, Zhang EB, De $W$ and Wang ZX. Downregulation of BRAF activated non-coding RNA is associated with poor prognosis for non-small cell lung cancer and promotes metastasis by affecting epithelial-mesenchymal transition. MOL CANCER. 2014; 13:68.

24. Birney E, Stamatoyannopoulos JA, Dutta A, Guigo R, Gingeras TR, Margulies EH, Weng Z, Snyder M, Dermitzakis ET, Thurman RE, Kuehn MS, Taylor CM, Neph S, Koch CM, Asthana S and Malhotra A, et al. Identification and analysis of functional elements in 1\% of the human genome by the ENCODE pilot project. NATURE. 2007; 447(7146):799-816.

25. Mattick JS. The central role of RNA in human development and cognition. FEBS LETT. 2011; 585(11):1600-1616.

26. Lee JT. Epigenetic regulation by long noncoding RNAs. SCIENCE. 2012; 338(6113):1435-1439.

27. Ponting CP, Oliver PL and Reik W. Evolution and functions of long noncoding RNAs. CELL. 2009; 136(4):629-641.

28. Eddy SR. Non-coding RNA genes and the modern RNA world. NAT REV GENET. 2001; 2(12):919-929.

29. Rossi MN and Antonangeli F. LncRNAs: New Players in Apoptosis Control. Int J Cell Biol. 2014; 2014:473857.

30. Qiu MT, Hu JW, Yin $\mathrm{R}$ and $\mathrm{Xu}$ L. Long noncoding RNA: an emerging paradigm of cancer research. Tumour Biol. 2013; 34(2):613-620.

31. Jiang $W$, Zhang D, Xu B, Wu Z, Liu S, Zhang L, Tian Y, Han X and Tian D. Long non-coding RNA BANCR promotes proliferation and migration of lung carcinoma via MAPK pathways. BIOMED PHARMACOTHER. 2015; 69:90-95.

32. Guo Q, Zhao Y, Chen J, Hu J, Wang S, Zhang D and Sun Y. BRAF-activated long non-coding RNA contributes to colorectal cancer migration by inducing epithelial-mesenchymal transition. ONCOL LETT. 2014; 8(2):869-875.

33. Wang $Y$, Guo Q, Zhao $Y$, Chen J, Wang S, Hu J and Sun Y. BRAF-activated long non-coding RNA contributes to cell proliferation and activates autophagy in papillary thyroid carcinoma. ONCOL LETT. 2014; 8(5):1947-1952

34. Roberts PJ and Der CJ. Targeting the Raf-MEK-ERK mitogen-activated protein kinase cascade for the treatment of cancer. ONCOGENE. 2007; 26(22):3291-3310.

35. Sathe A, Guerth F, Cronauer MV, Heck MM, Thalgott M, Gschwend JE, Retz $\mathrm{M}$ and Nawroth R. Mutant PIK3CA controls DUSP1-dependent ERK 1/2 activity to confer response to AKT target therapy. Br J Cancer. 2014; 111(11):2103-2113

36. Hou P, Liu D, Shan Y, Hu S, Studeman K, Condouris S, Wang Y, Trink A, El-Naggar AK, Tallini G, Vasko V and Xing M. Genetic alterations and their 
relationship in the phosphatidylinositol 3-kinase/Akt pathway in thyroid cancer. CLIN CANCER RES. 2007; 13(4):1161-1170.

37. Liu Z, Hou P, Ji M, Guan H, Studeman K, Jensen K, Vasko V, El-Naggar AK and Xing M. Highly prevalent genetic alterations in receptor tyrosine kinases and phosphatidylinositol 3-kinase/akt and mitogen-activated protein kinase pathways in anaplastic and follicular thyroid cancers. J Clin Endocrinol Metab. 2008; 93(8):3106-3116.

38. Xing M. Molecular pathogenesis and mechanisms of thyroid cancer. NAT REV CANCER. 2013; 13(3):184-199.

39. Nazarian R, Shi H, Wang $\mathrm{Q}$, Kong X, Koya RC, Lee H, Chen Z, Lee MK, Attar N, Sazegar H, Chodon T, Nelson SF, McArthur G, Sosman JA, Ribas A and Lo RS. Melanomas acquire resistance to B-RAF(V600E) inhibition by RTK or N-RAS upregulation. NATURE. 2010; 468(7326):973-977.

40. Johannessen CM, Boehm JS, Kim SY, Thomas SR, Wardwell L, Johnson LA, Emery CM, Stransky N, Cogdill AP, Barretina J, Caponigro G, Hieronymus H, Murray RR, Salehi-Ashtiani K, Hill DE and Vidal M, et al. COT drives resistance to RAF inhibition through MAP kinase pathway reactivation. NATURE. 2010; 468(7326):968-972.

41. Wagle N, Emery C, Berger MF, Davis MJ, Sawyer A, Pochanard P, Kehoe SM, Johannessen CM, Macconaill LE, Hahn WC, Meyerson M and Garraway LA. Dissecting therapeutic resistance to RAF inhibition in melanoma by tumor genomic profiling. J CLIN ONCOL. 2011; 29(22):3085-3096. 